\title{
Integration of Genetic Algorithm and Support Vector Machine to Predict Rail Track Degradation
}

\author{
Amir Falamarzi ${ }^{1}$, Sara Moridpour ${ }^{1}$, Majidreza Nazem ${ }^{1}$, Reyhaneh Hesami ${ }^{2}$ \\ ${ }^{1}$ Civil and Infrastructure Engineering Discipline, School of Engineering, RMIT University, Australia \\ ${ }^{2}$ Asset Planning and Visualisation, Yarra trams, Australia
}

\begin{abstract}
Gradual deviation in track gauge of tram systems resulted from tram traffic is unavoidable. Tram gauge deviation is considered as an important parameter in poor ride quality and the risk of train derailment. In order to decrease the potential problems associated with excessive gauge deviation, implementation of preventive maintenance activities is inevitable. Preventive maintenance operation is a key factor in development of sustainable rail transport infrastructure. Track degradation prediction modelling is the basic prerequisite for developing efficient preventive maintenance strategies of a tram system. In this study, the data sets of Melbourne tram network is used and straight rail tracks sections are examined. Two model types including plain Support Vector Machine (SVM) and SVM optimised by Genetic Algorithm (GASVM) have been applied to the case study data. Two assessment indexes including Mean Squared Error (MSE) and the coefficient of determination $\left(\mathrm{R}^{2}\right)$ are employed to evaluate the performance of the proposed models. Based on the results, GA-SVM model produces more accurate outcomes than plain SVM model.
\end{abstract}

\section{Introduction}

Nowadays public transport is regarded as an effective alternative to private transport. This mode of transportation has several advantages such as reducing road casualties, mitigating air and environmental pollution, easing traffic congestion and promoting healthy lifestyle $[1,2]$. Tram as a light rail transit is implemented in different places to facilitate the movement of people. As an accessible approach of public transport option, tram has different benefits compared to conventional transit systems. For example, they are more economically efficient due to lower cost of repair and purchase than heavy rail trains. Because of their design and lower weight, trams are able to travel on steeper gradients and tighter curves compared to conventional trains. In addition, compared to buses, they are more durable and able to carry more passengers. frams also provide more reliable services through the central business districts (CBD) than conventional buses. On the other hand, degradation of tram tracks can be increased because of population growth in big cities which leads to higher demand for the rail transport. In addition, because of sharing the routes with other private vehicles, trucks and buses, tram tracks undergo further pressure and stress $[3,4]$.

Based on the previous works, the degradation in tram tracks is usually measured in terms of track geometry defects including longitudinal level defects, the horizontal alignment defects, excessive gauge deviations, cross-level and track twist $[5,6]$. In order to design a sustainable rail planning and management system to mitigate the degradation of rail tracks, effective preventive maintenance operations and rehabilitation actions are required. Comparative analysis conducted for light rail transport demonstrated that designing strategies and programs for tram infrastructure maintenance is a multifaceted problem in which the behaviour modelling of track degradation is an essential part. Without developing rail track degradation prediction models, designing preventive maintenance strategies is not possible $[7,8]$.

This research intends to create rail track degradation models by using track gauge parameter data. Investigating several studies has shown that track gauge parameter can be employed as an effective representative of degradation of whole track infrastructure. This paper is organised as follows. Section 2 reviews the existing works on modelling of rail track degradation prediction. Section 3 presents the case study which consists of the data of Melbourne tram network over multiple years. Section 4 describes the development process of the proposed models including plain SVM and GA-SVM. Section 5 yields the results of the models and discussions. Finally, Section 6 presents the conclusion of this study.

\section{Literature review}

Several studies based on different methods have been carried out in the field of modelling of rail track degradation. In this study, as the establishment of SVM models are decided, the literature review presented below mainly considers this type of model. 
Asada et al. [9] elaborated an algorithm for developing fault detection of point machines and railway condition monitoring systems based on the SVM model. A point machine is a mechanical device used to turn the switch blades (point blades) from one point to another point in order to provide different routes for trains. In this research, two faults were addressed including overdriving and under-driving of the drive rod. Data collected from a Japanese AC point machine were applied. The results of system validation represented that the developed SVM model had accurate and valid performance in terms of classification of fault detection with different severity levels by using it in the electrical active power data of the point machine.

Ebrahimi et al. [10] elaborated SVM and a mechanistic model for prediction of ballast deformation of railway substructure in order to schedule the maintenance of ballast. Both models used the data collected from a large-scale cyclic tri-axial apparatus. Two distinguished phases were carried out including an initial compaction phase and a fouling impact phase. A gradual integration of plastic deformation of railway ballast in different fouling, traffic loading and moisture is used in conjunction with a subgrade deformation model to estimate the surface deviation of the railway track. Five-fold cross-validation was applied in this study. Based on the results of this study, the SVM method predicted the rate of plastic strain of ballast more accurately than the mechanistic model.

Cárdenas-Gallo et al. [11] conducted a research to classify different types of rail track defects such as alignment, profile and cross-level into yellow tag defects and red tag defects by applying SVM method. Red tag defects are defects in which the value of track geometry parameters exceed the track safety thresholds provided by Federal Railroad Administration (FRA) and must be fixed as soon as possible. Yellow tag defects satisfy FRA thresholds but they will ultimately turn into red tag defects if they are not treated. Capability to predict when yellow tag defects would progress toward red tags, assists railroads administrators in delivering more efficiently rail maintenance strategies. With respect to the result of the study, the performance of SVM model in classification of profile defects was successful.

\section{Case study}

In this research, Melbourne tram as the one of the largest tram networks in the world has been used as a case study. This network consists of $250 \mathrm{~km}$ of double tracks and 25 routes. Based on Yarra Trams (the operator of Melbourne tram network) reports [12], in 2017 Melbourne tram network provided service to more than 204 million passengers, which represented an annual increase of 0.2 percent. This data set includes a wide range of parameters such as track geometry parameters (e.g. profile, alignment, gauge and twist), annual traffic in MGT, rail profile, track surface, rail type, rail support, location and the year of instalment. This data set covers the years from 2009 to 2015 . For measuring track geometry parameters, a chord length of $10 \mathrm{~m}$ is used. As the presence of noisy and outliers in maintenance data sets is usual, data filtration technique has been applied to prepare the data set for development of the models. Data filtration is the procedure of removing noisy (out of range) and incomplete data from the main data set in order to improve the accuracy of the models. If the distribution of data set behaves like the normal distribution it can be considered that $99.7 \%$ of data are in the range of $3 \times \mathrm{SD}$ distance from the mean value [13, 14], where SD is the Standard Deviation. In this study, statistical tests such as Shapiro-Wilk test and Kolmogorov-Smirnov test have been employed. These tests are helpful measures to examine the possibility of the normal distribution. After analysing different track sections, it has been revealed that the changes in track gauge parameter follow a normal distribution. In this context, track sections in which gauge deviation parameters deviate from $\mu \pm 3 \times$ SD values are labelled as outlier data and are eliminated from the data set. In the final stage, the remaining data is controlled again and data with incomplete specifications are removed.

\section{Model development}

In this research, predicting track gauge deviation as a representative of tram rail degradation based on historical data has been studied. By reviewing the relevant works, input parameters including track gauge deviation in previous year (Gt-1), MGT, curve radii, rail support (RS), rail profile (RP), rail type (RT) and track surface (TS) are selected. The output parameter is track gauge in current year $(\mathrm{Gt})$.

In this research in attempt to represent a broader perspective on track degradation modelling, two models including plain SVM and GA-SVM have been applied.

In this research, as the target parameter is a continuous variable, $\mathrm{R}^{2}$ and MSE have been used to assess the performance of the proposed models as follows:

$$
R^{2}=1-\frac{\sum_{i=1}^{N}\left(y_{i}-f_{i}\right)^{2}}{\sum_{i=1}^{N}\left(y_{i}-\bar{y}\right)^{2}}
$$

where $R^{2}$ denotes the coefficient of determination and provides information about goodness of fit of the model, $N$ denotes the number of samples, $f_{i}$ denotes the value estimated by the model, $y_{i}$ represents the observed data and $\bar{y}$ is mean value of $y_{i}$.

$$
M S E=\frac{1}{N} \sum_{i=1}^{N}\left(y_{i}-f_{i}\right)^{2}
$$

In this study, R Statistical Software package is used to build the SVM and GA-SVM models. In the following sections, development process of SVM and GA-SVM are represented.

\subsection{SVM model}

SVM model can be categorised into two major classes including Support Vector Classification (SVC) and 
Support Vector Regression (SVR). SVR employs the adaptive margin-based loss functions and delivers the learning data (non-linear or linear) to higher dimensional feature space. This method explores the best possible decision rule with regards to generalisation ability [15, 16]. Regression estimation can be established by finding a function of $\mathrm{y}=\mathrm{f}(\mathrm{x})$ based on a set of training sample $\left\{X=\left(x_{i}, d_{i}\right), i=1, \ldots, N\right\}$, where $x_{i} \in R^{n}$ is the input vector (training data set), $d_{i}$ is the objective value of training sample and $N$ represents the number of training samples. To find the variables of the SVM model, the following optimisation problem needs to be solved:

$$
\begin{gathered}
\min \frac{1}{2} \sum_{i=1}^{N} \sum_{j=1}^{N}\left(\propto_{i}-\propto_{i}^{*}\right)\left(\propto_{j}-\propto_{j}^{*}\right) k\left(x_{i}, x_{j}\right) \\
+\varepsilon \sum_{i=1}^{N}\left(\propto_{i}+\propto_{i}^{*}\right) \\
-d \sum_{i=1}^{N}\left(\propto_{i}-\propto_{i}^{*}\right)
\end{gathered}
$$

s.t. $\sum_{i=1}^{N}\left(\propto_{i}-\propto_{i}^{*}\right)=0$ and $\propto_{i}, \propto_{i}^{*} \in[0, C]$

where $\propto_{i}$ and $\propto_{i}^{*}$ are Lagrange multipliers, $\varepsilon$ and $C$ are the hyper parameters and $k\left(x_{i}, x\right)$ denotes a definite kernel function.

Kernel function can be defined in different forms including Linear kernel function, Polynomial kernel function, Gaussian kernel function and Multilayer perception kernel [17]. In this study, Gaussian kernel function is used.

\subsection{GA-SVM}

GA is used in engineering to generate effective solutions for optimisation problems by simulating genetic evaluation process and applying bio-inspired operators such as crossover, mutation and selection. This mechanism commences from an initial population and by randomly continues evaluation (selecting, crossing and variation) from one generation to another generation, a group of highly-adaptive individual can be produced at last. Instead of finding local optimality, this algorithm has the ability to address global optimality $[18,19]$. In this study, by employing GA, the main parameters of SVM including $C$ and $\gamma$ are searched and optimised. Parameter $C$ governs the trade-off cost between minimising the complexity of the SVM model and the training error, whereas parameter $\gamma$ is not technically considered as a SVM parameter but, it is a parameter of the kernel function. In the RBF kernel, it determines how similar two points should be adjusted to be regarded similar. These two parameters have a strong influence on the generalisation performance and efficiency of the SVM model.

\section{Results and Discussion}

In this section, the results of the models with respect to their type are provided and discussed. Also, a comparison between the two models is presented.

First, the results of development of a plain SVM model for predicting the current track gauge deviation are presented in Table1. In this regard, the values of $\mathrm{R}^{2}$ and MSE of the SVM models for straight data sets are calculated and provided in Table 1 .

Table 1. Performance of SVM model

\begin{tabular}{|c|c|c|}
\hline Parameters & Adjusted $\mathbf{R}^{2}$ & MSE \\
\cline { 1 - 1 } $\mathrm{G}_{\mathrm{t}}$ & \multirow{2}{*}{0.90} & \multirow{2}{*}{1.95} \\
\cline { 1 - 1 } $\mathrm{R}_{\mathrm{T}}$ & & \\
\cline { 1 - 2 } $\mathrm{G}_{\mathrm{t}-1}$ & & \\
\hline
\end{tabular}

Figure 1 demonstrates the relationship between the data predicted by SVM model versus the observed data.

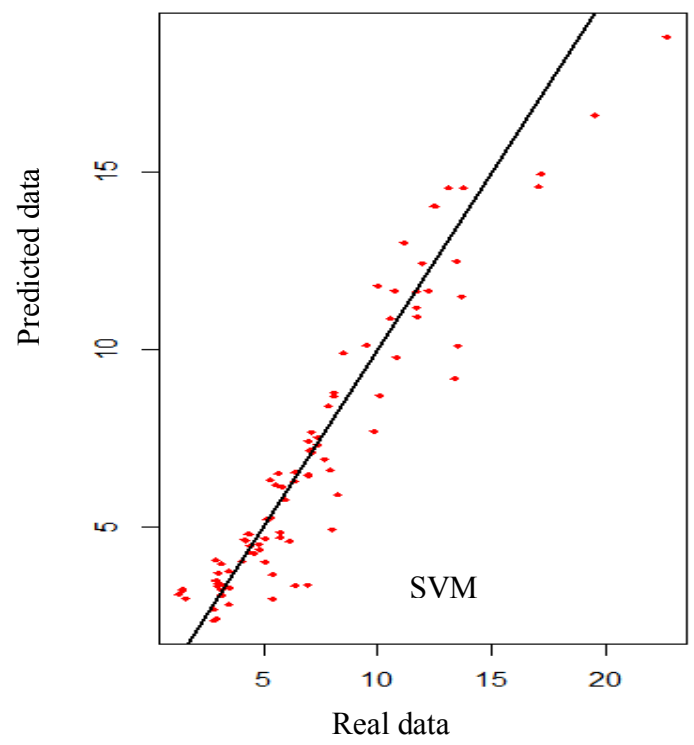

Figure 1. Observed data against predicted data (SVM)

Based on the results of the plain SVM models adjusted $\mathrm{R}^{2}$ is 0.90 and the values of MSE is 1.95 . In Table 2, the results of development of GA-SVM model for track gauge deviation prediction is presented.

Table 2. Performance of GA-SVM model

\begin{tabular}{|c|c|c|}
\hline Parameters & Adjusted $\mathbf{R}^{\mathbf{2}}$ & MSE \\
\cline { 1 - 1 } $\mathrm{G}_{\mathrm{t}}$ & & \\
\cline { 1 - 1 } $\mathrm{R}_{\mathrm{T}}$ & 0.92 & 1.60 \\
\cline { 1 - 2 } $\mathrm{G}_{\mathrm{t}-1}$ & & \\
\hline
\end{tabular}

Based on the results of the GA-SVM model, adjusted $R^{2}$ is 0.92 and the values of MSE is 1.60 . In Figure 2, the relationship between GA-SVM predicted data against observed data is shown. 


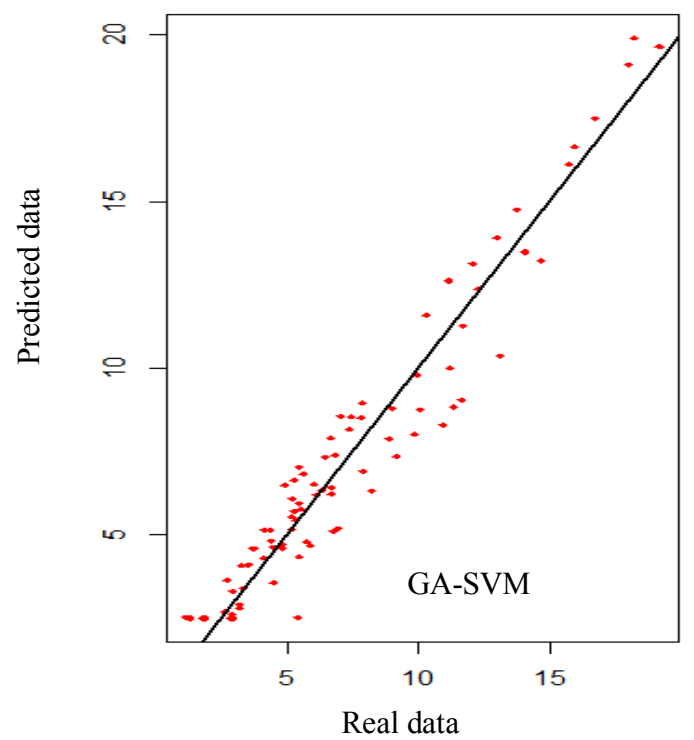

Figure 2. Observed data against predicted data (GA-SVM)

The results, which agree with the previous research works $[20,21,22]$, show the strong correspondence between initial value of the track geometry parameters and current value of the parameters as well as rail type. Based on the results derived from the proposed models and in terms of adjusted $\mathrm{R}^{2}$ and MSE, GA-SVM model represents a better performance than the plain SVM model in prediction of gauge deviation. These results are shown in Figures $1 \& 2$ where the predicted data of GA-SVM model are closer to the observed data.

\section{Conclusion}

Track gauge deviation is represented as a valuable measure of ride quality and the potential of train derailment. Practices for predication of gauge deviation is a principal prerequisite in establishment of the preventive maintenance operations. Performing preventive maintenance operations which is considered as an essential part of tram infrastructure maintenance and management systems, will result in reliable tram services along with decrease in rail maintenance cost. In this research, track data collected from Melbourne tram network was applied as a case study. Data used in this study composed of different parameters but the key parameters included track gauge deviation along with traffic data and structural parameters.

In this research for track degradation prediction, two AI approaches including SVM and GA-SVM models were used and developed. Along with model development, models were assessed based on MSE and the coefficient of determination. According to the results of this study, the performance of both SVM and GA-SVM models in predicting gauge deviation values of straight track sections is satisfying. In this regards, the values of $\mathrm{R}^{2}$ for both models are above 0.90 and MSE values are less than 2.00. The results also showed that due to optimisation of the parameters of SVM model, the GA-SVM model is more accurate in prediction of track gauge than the plain SVM model.

\section{References}

1. Redman, L., Friman, M., Gärling, T., \& Hartig, T. (2013). Quality attributes of public transport that attract car users: A research review. Transport Policy, 25, 119127.

2. D. Knowles, R., \& Ferbrache, F. (2016). Evaluation of wider economic impacts of light rail investment on cities. Journal of Transport Geography, 54, 430-439.

3. Gadziński, J., \& Radzimski, A. (2016). The first rapid tram line in Poland: How has it affected travel behaviours, housing choices and satisfaction, and apartment prices? Journal of Transport Geography, 54, 451-463.

4. Ahac, M., \& Lakusic, S. (2017). Track Gauge Degradation Modelling on Small Urban Rail Networks: Zagreb Tram System Case Study. Urban Transport Systems, (January).

5. He, Q., Li, H., Bhattacharjya, D., Parikh, D. P., \& Hampapur, A. (2015). Track geometry defect rectification based on track deterioration modelling and derailment risk assessment. Journal of the Operational Research Society, 66(3), 392-404.

6. Andrade, A. R., \& Teixeira, P. F. (2018). Assessing Temporary Speed Restrictions and Associated Unavailability Costs in Railway Infrastructure. International Journal of Civil Engineering, 16(2), 219228.

7. Andrews, J. (2012). A modelling approach to railway track asset management. Proceedings of the Institution of Mechanical Engineers, Part F: Journal of Rail and Rapid Transit, 227(1), 56-73.

8. An, R., Sun, Q., Wang, F., Bai, W., Zhu, X., \& Liu, R. (2018). Improved Railway Track Geometry Degradation Modeling for Tamping Cycle Prediction, 144(2015), 1-11.

9. Asada, T., Roberts, C., \& Koseki, T. (2013). An algorithm for improved performance of railway condition monitoring equipment: Alternating-current point machine case study. Transportation Research Part C: Emerging Technologies, 30, 81-92.

10. Ebrahimi, A., Tinjum, J. M., \& Edil, T. B. (2014). Maintenance model for railway substructure. Geotechnical Engineering, 45(1), 48-55.

11. Cárdenas-Gallo, I., Sarmiento, C. A., Morales, G. A., Bolivar, M. A., \& Akhavan-Tabatabaei, R. (2017). An ensemble classifier to predict track geometry degradation. Reliability Engineering and System Safety, 161(April 2016), 53-60.

12. PTV. (2017). Public Transport Victoria Annual Report. Melbourne. 
13. Grafarend, E. W. (2006). Linear and Nonlinear Models : Fixed Effects, Random Effects, and Mixed Models. Berlin: De Gruyter.

14. Gordon, S. (2006). The Normal Distribution. University of Sydney. Sydney.

15. Jiang, X., Zhang, L., \& Chen, M. X. (2014). Shortterm forecasting of high-speed rail demand: A hybrid approach combining ensemble empirical mode decomposition and gray support vector machine with real-world applications in China. Transportation Research Part C: Emerging Technologies, 44, 110-127.

16. Li, Q., Shi, Z., Zhang, H., Tan, Y., Ren, S., Dai, P., \& Li, W. (2018). A cyber-enabled visual inspection system for rail corrugation. Future Generation Computer Systems, 79, 374-382.

17. Ben-hur, A., \& Weston, J. (2010). Data Mining Techniques for the Life Sciences, 609, 223-239.

18. Li, X. Z., \& Kong, J. M. (2014). Application of GASVM method with parameter optimization for landslide development prediction. Natural Hazards and Earth System Sciences, 14(3), 525-533.

19. Zhang, Z., Qin, Y., Cheng, X., Zhu, L., Kou, L., Li, J., \& Sun, F. (2015). Metro Station Safety Status Prediction Based on GA-SVR. In International Conference on Electrical and Information Technologies for Rail Transportation (pp. 57-69). Berlin: Springer.

20. Andrade, A. R., \& Teixeira, P. F. (2015). Statistical modelling of railway track geometry degradation using Hierarchical Bayesian models. Reliability Engineering and System Safety, 142, 169-183.

21. Moridpour, S., Mazloumi, E., \& Hesami, R. (2017). Application of Artificial Neural Networks in Predicting the Degradation of Tram Tracks Using Maintenance Data. In Applied Big Data Analytics in Operations Management (pp. 30-54). Delhi, India: IGI Global.

22. Falamarzi, A., Moridpour, S., Nazem, M., \& Hesami, R. (2018). Rail Degradation Prediction Models for Tram System: Melbourne Case Study. Journal of Advanced Transportation, 2018, 8. 